\title{
Familias uruguayas con maltrato infantil: estresores y apoyo social en contexto de pobreza
}

\author{
María Eugenia Fernández Rodríguez y Cecilia Valentina Cracco Cattani ${ }^{2}$ \\ Universidad Católica de Uruguay-Uruguay
}

\begin{abstract}
El objetivo de este trabajo ha sido caracterizar a familias con presencia de maltrato infantil y abuso sexual en situación socioeconómica deficitaria en relación con estresores familiares y apoyo social, y analizar sus implicancias para las intervenciones psicosociales. La muestra estuvo conformada por 40 adultos a cargo del cuidado de nińos, niñas y adolescentes que asisten a servicios especializados en maltrato infantil en Uruguay. Los resultados indicaron graves situaciones de vulnerabilidad socioeconómica, feminización de cuidados, polivictimización de los niños, alto número de estresores y baja percepción de apoyo social. Se concluye en la importancia de considerar estas variables al momento de diseñar intervenciones psicosociales que propongan cambios a nivel de parentalidad bien tratante que puedan ser sostenidos en el tiempo.

Palabras clave: maltrato infantil, estresores familiares, apoyo social, pobreza, intervenciones psicosociales.
\end{abstract}

\section{Childhood at risk: child abuse, family stressors, social support and poverty}

The aim of this paper is to describe a group of families of a low socio-economic status in which child abuse and sexual abuse has been identified, linking such infringement of children rights with family stressors and social support to analyze the implications such link has for psychosocial interventions. The sample was composed by 40 adults who had children and adolescents under their care who receive help from services specialized in child abuse in Uruguay. It was found that the families studied had high socioeconomic vulnerability, childcare mainly done by women, children who had experienced polyvictimization, a high number of stressors and a low perception of social support. Conclusions drawn outline the importance of considering these variables when designing intervention programs that focus on promoting changes in parental practices that can be long lasting.

Keywords: child abuse, family stressors, social support, poverty, psychosocial interventions.

1 Magíster en Familias y Psicología Sistémica. Investigadora honoraria en la Universidad Católica de Uruguay. Dirección postal: Av. 8 de Octubre 2738, 11600 Montevideo, Departamento de Montevideo, Uruguay. Contacto: eugenia.f.1991@gmail.com. https://orcid. org/0000-0002-3594-885X

2 Doctora en Psicología. Directora del Departamento de Psicología Clínica y de la Salud en la Universidad Católica del Uruguay. Dirección postal: Av. 8 de Octubre 2738, 11600 Montevideo, Departamento de Montevideo, Uruguay. ccracco@ucu.edu.uy. https://orcid. org/0000-0002-3594-885X 
Infância vulnerável: abuso infantil, estressores familiares, suporte social e pobreza

O objetivo deste trabalho foi caracterizar famílias com presença de maus-tratos e abuso sexual infantil em situação socioeconômica deficitária em relação aos estressores familiares e suporte social, também analisar suas implicaçóes para intervençóes psicossociais. A amostra foi constituída por 40 adultos encarregados de crianças e adolescentes abastecidos em serviços especializados em maus-tratos infantis no Uruguai. Os resultados indicaram graves situaçôes de vulnerabilidade socioeconômica, feminização dos cuidados, múltipla vitimização de crianças, elevado número de estressores e baixa percepçáo de suporte social. Concluímos sobre a importância dessas variáveis ao projetar intervençóes psicossociais que proponham mudanças no nível do tratamento parental que podem ser sustentadas ao longo do tempo.

Palavras-chave: abuso infantil, estressores familiares, suporte social, pobreza, intervençóes psicossociais.

Enfance à risque: altraitance d'enfants, facteurs de stress familiaux, soutien social et pauvreté

L'objectif de ce travail a été de caractériser les familles en présence de maltraitance et d'abus sexuels sur des enfants en situation socio-économique déficitaire par rapport aux stresseurs familiaux et au soutien social, et d'analyser leurs implications pour les interventions psychosociales. L'échantillon était composé de 40 adultes en charge de la prise en charge des enfants et adolescents qui fréquentent des services spécialisés dans la maltraitance des enfants en Uruguay. Les résultats ont indiqué des situations graves de vulnérabilité socioéconomique, de féminisation des soins, de victimisation multiple des enfants, un nombre élevé de facteurs de stress et une faible perception du soutien social. Il est conclut sur l'importance de prendre en compte ces variables lors de la conception d'interventions psychosociales qui proposent des changements au niveau de la parentalité bien traitant qui peuvent être soutenus dans le temps.

Mots-clés: maltraitance des enfants, facteurs de stress familiaux, soutien social, pauvreté, interventions psychosociales. 
El maltrato infantil es un problema de agenda pública, de derechos humanos y de salud, que afecta negativamente la vida de muchos niños, nińas y adolescentes (NNA) a nivel mundial. Cada año se detectan cerca de mil millones de NNA afectados por algún tipo de maltrato (físico, sexual o psicológico); 300 millones tienen entre 2 y 4 años de edad (Organización de las Naciones Unidas, 2020). A partir del reconocimiento de este fenómeno como un problema, se ha legislado para garantizar la protección infantil a nivel internacional, nacional, comunitario y familiar. El Estado uruguayo asume compromisos internacionales de velar por la seguridad de los NNA e intervenir en las situaciones de vulneración de sus derechos (Ley $\mathrm{N}^{\circ}$ 17.823, 2004; Convención de los Derechos del Nińos, 1989).

Uruguay presenta cifras alarmantes de violencia infantil, que en los últimos años han incrementado significativamente. Según el Sistema Integral de Protección a la Infancia y Adolescencia contra la Violencia (SIPIAV) durante 2019 se detectaron 4774 NNA víctimas de violencia a nivel nacional, dando cuenta de un aumento cuantitativo en la detección superior a cinco veces en los últimos 8 años de registro. De esos NNA, 32\% fue maltratado emocionalmente, $24 \%$ sexualmente, $22 \%$ físicamente y $22 \%$ ha sido víctima de negligencia. El $91 \%$ de los/as agresores/as son familiares de los NNA (SIPIAV, 2020).

El maltrato infantil atraviesa todos los sectores de la sociedad, no excluye sexos, tipos de familias, tampoco niveles socioeconómicos, constituyéndose en un grave factor de riesgo para el desarrollo (Bentancor et al., 2017; Grinberg, 2015). Sin embargo, el contexto socioeconómico vulnerable conlleva complejidades que deben ser especialmente consideradas.

Las familias que viven en contextos críticos presentan acumulación de demandas que elevan sus niveles de estrés. Los modelos de estrés y vulnerabilidad familiar (Conger et al., 1992) han demostrado 
empíricamente que la insuficiencia de recursos económicos se asocia a estrés económico percibido y que este afecta la salud mental de los adultos incidiendo negativamente en la calidad de los vínculos conyugales y en las prácticas parentales que se ven empobrecidas (Bartholomae y Fox, 2017; Conger et al., 2010; Kiernan y Huerta, 2008; Leinonen et al., 2002; Masarik y Conger, 2017; Newland et al., 2013). Si bien no en todos los casos estas prácticas implican violencia, se plantea que los adultos de las familias en contextos de pobreza desarrollan prácticas de crianza más inconsistentes, focalizadas en la obediencia y en la inhibición inmediata de la conducta (Hill, 2012).

Los niveles de estrés dependen de los recursos que disponga el sistema familiar, pero cuando las demandas son excesivas, los recursos se van agotando y las posibilidades de respuestas eficientes se van reduciendo, conduciendo a un efecto de acumulación de estresores que deteriora la capacidad del sistema familiar para cumplir con sus funciones (Brooks-Gunn et al., 2013; Cabrera et al., 2012).

Khaw (2017) plantea que el estrés crónico es característico de las familias con situaciones de violencia intrafamiliar. Las familias estresadas y con funcionamiento violento disponen de menor apoyo social (Musitu y Cava, 2001). Además, suelen presentar dinámicas caóticas o rígidas, con fronteras extremadamente impermeables o difusas. Generalmente se trata de generaciones de ausencia de cuidados, necesidades básicas insatisfechas, e inserción en redes comunitarias empobrecidas y/o disfuncionales, con altas demandas, problemas y crisis recurrentes.

En este escenario queda poco espacio para la reflexión y gestión de soluciones, absorbiendo a los servicios sociales en una lógica donde se termina actuando en la emergencia de lo cotidiano sin lograr afectar las estructuras familiares para lograr cambios reales y perdurables. El poco éxito de las intervenciones psicosociales lleva en muchos casos a la multi-intervención que se suma a la espiral de vulnerabilidades acumuladas (Cleek et al., 2012; Minuchin et al., 2000; Musitu y Cava, 2001).

Considerando las asociaciones reportadas en la literatura entre estrés familiar, violencia intrafamiliar, apoyo social y nivel socioeconómico deficitario, este trabajo tiene como objetivo caracterizar a familias 
uruguayas con maltrato infantil en relación con estas variables y analizar las posibles implicancias para las intervenciones psicosociales. Se plantean como objetivos específicos: a) describir a las familias participantes con relación a variables sociodemográficas, b) describir cantidad y tipo de estresores que reportan los adultos a cargo de NNA, c) describir el apoyo social reportado por los adultos a cargo, y d) analizar la relación entre estresores y el apoyo social de las familias con maltrato hacia NNA.

\section{Método}

\section{Participantes}

La muestra se conformó por 40 referentes familiares adultos a cargo del cuidado de NNA que iniciaron procesos psicosociales en una Organización No Gubernamental (ONG) especializada en maltrato infantil durante los meses de febrero a noviembre de 2019, en dos ciudades de Uruguay. Estos casos representan el 93\% de las familias que asistieron a los servicios en ese periodo. Por las características de la ONG, acceden a atención familias con niveles socioeconómicos deficitarios que son derivadas por instituciones que detectan situaciones de vulneraciones de derechos graves. El 87.5\% de los referentes son de sexo femenino y el $12.5 \%$ masculino, con una edad media de $36.2(D E=12.9)$ y que están a cargo del cuidado de 116 NNA $(M=2.3$ NNA por adulto). El $72.5 \%$ de la muestra se encuentra conformada por madres, $12.5 \%$ por abuelas/os, $7.5 \%$ por padres, $5 \%$ parejas de padres y $2.5 \%$ otros.

\section{Instrumentos}

Cuestionario sociodemográfico: diseñado ad hoc para consignar motivo de atención (tipo de violencia), número de agresores (vínculo con NNA), edad del adulto que responde, relación de parentesco con el NNA, estado civil, nivel educativo de la madre y del padre, situación de empleo y configuración familiar. 
Índice de Nivel Socioeconómico (INSE; Perera, 2018): estima puntos de corte que definen los niveles socioeconómicos según la capacidad de consumo del sistema familiar. Se clasifican los niveles socioeconómicos en bajo (B), medio (M) y alto (A), compuestos por siete subniveles que distinguen: $\mathrm{B}-\mathrm{y} \mathrm{B}+$; M-, $\mathrm{M}$ y M+; A- y A+.

Family Inventory of Life Events and Changes (FILE; McCubbin et al., 1989; versión de Hernández-Córdoba, 1991), valora la acumulación de estresores experimentados por una familia en los últimos 12 meses. Consta de 80 ítems que se agrupan en 9 categorías: 1) tensiones intrafamiliares, 2) tensiones maritales, 3) tensiones del embarazo y crianza, 4) tensiones económicas y de negocios, 5) tensiones y transiciones laborales, 6) enfermedad y tensiones del cuidado familiar, 7) pérdidas, 8) transiciones por entrada y salida de miembros del hogar, y 9) legales. A cada respuesta "SÎ" se le asigna 1 punto, a mayor puntaje, mayor número de estresores experimentados. Se administró la versión utilizada por Cracco (2017) que cuenta con ajustes lingüísticos a la población uruguaya. Los índices de fiabilidad $\alpha$ de Cronbach reportados en su estudio $(N=239)$ fueron de .86 para la escala global, .74 para las tensiones Intrafamiliares, .62 para Maritales, .42 para Embarazo, .56 para Económicas, .46 para Laborales, .44 para Enfermedad, .45 para Pérdidas, .30 para Transiciones, y .63 para Ley. A este instrumento se le agregó al final el ítem "Otros sucesos estresantes", donde se tuvo en cuenta otros estresores que pudieran estar afectando a los adultos y que no estuvieron contemplados en el inventario.

The MOS Social Support Survey (Sherbourne y Stewart, 1991): creado originalmente en Estados Unidos para medir el apoyo social en pacientes con enfermedades crónicas. Se utilizó la versión validada en Argentina por Rodríguez y Enrique (2007). El instrumento se compone por 20 ítems. El primer ítem valora el apoyo estructural que refiere a características cuantitativas y objetivas de la red de apoyo social (tamaño y densidad) y se responde mencionando cuántas personas le brindan apoyo. Los 19 ítems restantes evalúan el apoyo funcional percibido, preguntando con qué frecuencia se dispone de cada uno de los apoyos, y se responden a través de una escala Likert de 5 puntos 
(nunca, pocas veces, algunas veces, la mayoría de las veces o siempre). En la versión validada en Argentina el análisis factorial del apoyo funcional reveló la existencia de ttrd factores: apoyo emocional, instrumental e informacional (suprimen la interacción social positiva, cuarto factor contemplado en la versión original). El apoyo emocional refiere a sentimientos de ser querido, de seguridad, de confianza en alguien y de tener intimidad con esa persona; el apoyo instrumental es la posibilidad de disponer de ayuda directa cuando lo necesita; y el apoyo informacional consiste en la provisión de consejos o guía para ayudar a resolver sus problemas. Estos tres factores explicaron el $59.86 \%$ de la varianza global. La escala presentó un índice de consistencia interna $\alpha$ de Cronbach de .92 total, .88 para apoyo emocional, .84 para apoyo afectivo, y .80 para apoyo instrumental (Rodríguez y Enrique, 2007).

\section{Procedimiento}

Para conformar la muestra del estudio se contó con la autorización institucional de una ONG dedicada al trabajo con NNA víctimas de maltrato infantil y abuso sexual. Las administraciones fueron realizadas por la investigadora principal en forma individual y guiada, dadas las dificultades en lectura y/o comprensión lectora en los adultos participantes. Los instrumentos fueron administrados en la etapa de ingreso a los servicios de atención (primeros cinco encuentros) con una duración aproximada de 30 minutos. Antes de su administración, se solicitó la firma del consentimiento informado. El procedimiento, consentimientos y protocolos cuentan con el aval del Comité de Ética en la Investigación de la Universidad Católica del Uruguay, cumpliendo las normativas del país sobre investigación con seres humanos reguladas por el Decreto 001-4573/2007 del Poder Ejecutivo y de la Ley Nro. 18331 de Habeas Data, referida a la reserva de datos personales.

\section{Diseño y análisis de datos}

Se diseñó un estudio descriptivo, correlacional, de corte transversal (Montero y León, 2005). Se realizaron estadísticos descriptivos y 
correlaciones. En función de los resultados de los análisis de normalidad de las variables obtenidos mediante la prueba de Kolgomorov-Smirnov, se utilizaron pruebas paramétricas. Los cálculos se realizaron con el paquete estadístico SPSS (Statistical Package for the Social Sciences) versión 25 .

\section{Resultados}

\section{Características sociodemográficas de las familias}

El $45 \%$ de las familias que componen la muestra presenta una configuración monoparental (89\% con jefatura femenina y $11 \%$ con jefatura masculina), el $27.5 \%$ son familias extensas, el $20 \%$ ensambladas y el $7.5 \%$ nucleares.

En cuanto al nivel socioeconómico, 92.5\% de la muestra se encuentra en los niveles socioeconómico muy bajo y bajo $(77.5 \%$ B- y $15 \% \mathrm{~B}+$ ) y el restante en el nivel socioeconómico medio bajo (M-).

El 55\% de las madres cuenta con primaria, el 37.5\% con ciclo básico, el $7.5 \%$ con bachillerato. El 57.5\% de los padres realizó primaria, el $37.5 \%$ ciclo básico y el restante bachillerato y "otros" (formación técnica).

Sobre la situación de empleo, el 32.5\% refiere contar con empleo estable, $27.5 \%$ con empleo inestable, $22.5 \%$ en situación de desempleo, $10 \%$ son amas de casa y $7.5 \%$ en situación "otros" (jubilados o pensionistas). En el 10\% de los hogares no hay perceptor de ingresos, el 55\% de los hogares cuenta con un perceptor, el 30\% con dos, $2.5 \%$ con tres y $2.5 \%$ con cinco. El 30\% de las familias cuenta con prestaciones sociales como su único ingreso de subsistencia.

En cuanto a la situación habitacional, el 50\% respondió ser propietario de la vivienda donde reside; el 23\% han accedido a la vivienda mediante compra irregular, siendo un $27 \%$ los que efectivamente son propietarios (algunas edificadas irregularmente en terrenos de familiares). El $62.5 \%$ de las viviendas cuentan con techo de material precario y el $12.5 \%$ no posee baño. 
En cuanto al motivo de derivación y atención en los servicios, los NNA han vivenciado un mínimo de 2 tipologías de maltrato y un máximo de 6 (maltrato físico, emocional, sexual, químico, negligencia, violencia de género y violencia patrimonial). En la Tabla 1 se presenta la distribución de NNA en función del número de malos tratos recibidos a lo largo de sus vidas.

\section{Tabla 1}

Motivo de atención de los NNA

\begin{tabular}{lc}
\hline No de tipologías & $\%$ NNA \\
\hline 2 tipologías & 12.5 \\
3 tipologías & 25 \\
4 tipologías & 25 \\
5 tipologías & 17.5 \\
6 tipologías & 20 \\
\hline
\end{tabular}

El 100\% de los NNA han sido maltratados emocionalmente. En segundo lugar, se encuentra la violencia patrimonial afectando al $77.5 \%$ de los NNA, el abuso sexual con un 75\%, la violencia de género con un $67.7 \%$, el maltrato físico con un $60 \%$, la negligencia con un $27.5 \%$ y el $12.5 \%$ con la categoría "otros" que engloba violencia química e institucional.

En cuanto al número de agresores por NNA, en $22.5 \%$ de los casos se detecta un agresor, en $40 \%$ dos, en $32.5 \%$ tres y en $5 \%$ cuatro agresores. El 100\% de los NNA han sido agredidos por un familiar directo. El 40\% de los adultos que participaron del estudio ejercía algún tipo de maltrato hacia los NNA a su cargo. Ninguno ejerce abuso sexual ya que es requisito de ingreso al Servicio que el NNA se encuentre separado del agresor sexual antes de ser derivado (esta medida es realizada a nivel Judicial). 


\section{Cantidad y tipo de estresores reportados}

Se obtuvo una media de 16.8 estresores para el total de la muestra $(D E=6.8)$ puntuando un mínimo de 6 estresores y un máximo de 36 en una escala de 0 a 80 puntos.

Más del 90\% de los participantes presenta entre 11 y 30 estresores del listado en el último año de vida. En la Tabla 2 se presentan la media y el desvío estándar para las distintas categorías de estresores.

\section{Tabla 2}

Datos descriptivos de los estresores familiares

\begin{tabular}{lcl}
\hline Estresores & $M$ & $D E$ \\
\hline Estresores intrafamiliares & 5.0 & 3.3 \\
Estresores maritales & 1.3 & 1.1 \\
Estresores por embarazo & 1.1 & 0.7 \\
Estresores económicos & 2.5 & 1.8 \\
Estresores laborales & 2.2 & 1.4 \\
Estresores por enfermedades & 1.6 & 1.1 \\
Estresores por pérdidas & 1.2 & 0.7 \\
Estresores por transiciones & 1.3 & 0.6 \\
Estresores vinculados a la ley & 1.6 & 1.4 \\
Total de Estresores & 16.8 & 6.8 \\
\hline
\end{tabular}

Los estresores con mayor presencia en el último año corresponden a la categoría "tensiones intrafamiliares", seguidas por las "tensiones económicas" y "tensiones laborales" (ver Tabla 3).

Al final del inventario se agregó la opción "Otros", en donde se registraron respuestas que refieren a: "situación de desempleo", "situación económica", "dificultades en la puesta de limites a hijos", "situación habitacional", "inoperancia del poder judicial"y "sobrecarga de cuidados". 
Familias uruguayas con maltrato infantil / Fernández y Cracco

\section{Tabla 3}

Estresores familiares más reportados (en porcentajes)

\begin{tabular}{|c|c|}
\hline Estresores Intrafamiliares & $\%$ \\
\hline Aumentó el tiempo de la mujer fuera de la familia & 50 \\
\hline Uno de los miembros presentó problemas emocionales & 80 \\
\hline Uno de los hijos tuvo problemas de aprendizaje & 60 \\
\hline Aumentó el conflicto entre los hijos & 57.5 \\
\hline $\begin{array}{l}\text { Aumentaron las dificultades para manejar a los hijos en edad escolar ( } 6 \text { a } 12 \\
\text { ańos) }\end{array}$ & 50 \\
\hline Aumentaron las "actividades fuera de la casa" en la que participan los hijos & 50 \\
\hline \multicolumn{2}{|l|}{ Estresores económicos } \\
\hline Se tomó o se refinanció un préstamo para cubrir un aumento de gastos & 45 \\
\hline La familia se quedó sin ningún ingreso estable & 55 \\
\hline Aumentaron los gastos de comida, ropa, servicios, etc. & 77.5 \\
\hline $\begin{array}{l}\text { Demoras en recibir el aporte económico para el sostenimiento de los hijos } \\
\text { por parte del padre ausente o separado }\end{array}$ & 52.5 \\
\hline \multicolumn{2}{|l|}{ Estresores laborales } \\
\hline Uno de los miembros perdió o dejó el trabajo & 55 \\
\hline $\begin{array}{l}\text { Uno de los miembros comenzó o volvió al trabajo después de un largo } \\
\text { tiempo }\end{array}$ & 35 \\
\hline $\begin{array}{l}\text { Uno de los miembros dejó de trabajar por un largo período (por sanción, } \\
\text { huelga, licencia) }\end{array}$ & 32.5 \\
\hline Uno de los miembros adolescentes cambió de escuela o liceo & 42.5 \\
\hline
\end{tabular}

\section{Apoyo social percibido}

En la Tabla 4 se presentan los resultados descriptivos del apoyo social estructural reportados por los referentes. Este dato se obtuvo preguntando con cuántos familiares y amigos contaban. El 87.5\% de los adultos cuentan con menos de cinco personas. 


\section{Tabla 4}

Datos descriptivos del apoyo social estructural

\begin{tabular}{lll}
\hline & $M$ & $D E$ \\
\hline Familia & 1.4 & 1.8 \\
Amigos & 1.7 & 3.4 \\
Total & 3.1 & 5.2 \\
\hline
\end{tabular}

En la Tabla 5 se presentan los datos reportados para cada uno de los apoyos sociales funcionales.

\section{Tabla 5}

Datos descriptivos del apoyo social funcional

\begin{tabular}{lccc}
\hline Factores & $M$ & $D E$ & Rango \\
\hline Apoyo emocional/informacional & 25.4 & 12 & 10 a 50 \\
Apoyo afectivo & 18.2 & 5.4 & 5 a 25 \\
Apoyo instrumental & 9.5 & 5.2 & 4 a 20 \\
Apoyo total & 53.7 & 19.7 & 19 a 95 \\
\hline
\end{tabular}

\section{Relación entre estrés y apoyo social percibido}

Antes de realizar los estudios correlacionales, se analizó el tipo de distribución de las variables. Según los resultados del estadístico Kolmogorov-Smirnov $K-S$ para las variables de total de estresores y apoyo social, no es posible rechazar la hipótesis nula de distribución normal de las variables $(p>.05)$, por lo cual se realizaron cálculos de correlaciones bivariadas de Pearson (prueba paramétrica).

En cuanto al total de estresores y total de apoyo social, se encontró una correlación significativa y negativa entre ambas $(-.344 ; p=.030)$. Se realizó un análisis correlacional de todas las subcategorías que conforman el reporte de estresores y de apoyo social. De todas las correlaciones solamente fueron significativas las correlaciones entre apoyo social afectivo y violaciones a la ley $(-.319 ; p=.045)$, apoyo social ins- 
trumental y tensiones intrafamiliares $(-.372 ; p=.018)$, y apoyo social instrumental y pérdidas $(-.401 ; p=.001)$.

\section{Discusión}

Los resultados del trabajo muestran la alta vulnerabilidad psicosocial de las familias participantes. Respecto a los datos sociodemográficos, el porcentaje de familias monoparentales con jefatura femenina supera en cuatro veces al reportado a nivel nacional en el último censo (Cabella et al., 2015). Este tipo de configuración ha recibido especial atención por parte de la literatura (Waldfogel et al., 2010) destacando cómo la presencia de un solo adulto implica menores capacidades para generar ingresos y/o movilizar redes familiares o comunitarias (Kaztman y Rodríguez, 2005) siendo una única persona la que debe sostener económicamente el hogar y cumplir con las funciones de crianza.

Este tipo de familias se asocian a menores oportunidades de empleo, mayores dificultades de conciliación laboral, acceso a empleos en situación de precariedad con pocas garantías de derechos y protección social, redes de apoyo insuficientes y sobrecarga de cuidados (Sastre, 2015).

Los datos reportados por las familias muestran la feminización de los cuidados y ausencia de cumplimiento de las obligaciones paternas, con un $77.5 \%$ de violencia patrimonial. La feminización de cuidados no es un fenómeno aislado o intrínseco a las familias con violencia; se encuentra instalado en el imaginario social, en prácticas de crianzas, leyes y las políticas sociales (Batthyány et al., 2015; Cabella et al., 2015). Los cuidados siguen siendo una categoría identitaria y asumida por las mujeres, con costos asociados a la pérdida de autonomía, disminución en actividades remuneradas, bajos ingresos, dificultades en acceso a derechos sociales, exclusión, escaso goce de tiempo libre y descanso (Carrasco et al., 2011; García et al., 2004).

La ausencia de apoyo en los cuidados en situaciones de violencia familiar impacta en diferentes dimensiones; puede retraer la inserción laboral de mujeres y su independencia, incrementar la dependencia 
hacia los agresores, limitar posibilidades de salida o retorno al circuito violento (Casique, 2010).

Los tipos de violencias, el número de agresores reportado y el vínculo con los agresores, dan cuenta del riesgo en el que se encuentran las víctimas y delimitan las posibilidades de trabajo en su protección, reparación del daño psíquico y restitución de derechos (Intebi, 2009). Importa consignar aquí que los datos oficiales presentados por el SIPIAV se recogen de manera tal que solo es posible consignar un tipo de violencia sufrida por los NNA. En este trabajo, todos los NNA son víctimas de al menos dos tipos de violencia. La polivictimización se asocia a mayor riesgo de daño físico y mental duradero, destacándose especialmente la sintomatología asociada al trauma complejo (Finkelhor et al., 2011).

El alto número de estresores reportados por las familias participantes está en concordancia con datos planteados en otras investigaciones nacionales e internacionales con familias en contextos críticos (Brooks-Gunn et al., 2013; Cabrera et al., 2012; Capano y Pacheco, 2014; Cracco, 2017; Cracco y Blanco, 2015). Las categorías que englobaron a los estresores más reportados por las familias son concordantes con lo encontrado en familias con hijos en edad escolar y/o adolescente del Uruguay (Cracco, 2017; Cracco y Blanco, 2015), en estudios que muestran que los tipos de estresores no son distintos según el nivel socioeconómico de las familias, pero sí lo es la cantidad de estresores.

La acumulación de estresores y la situación de estrés crónico que supone la pobreza implica el desgaste de recursos con la consecuente dificultad para poner en marcha acciones de afrontamiento efectivo, llevando al desarrollo de estrategias de afrontamiento pasivas, emocionales o evasivas (Boss, 2002). Los niños criados en tales situaciones aprenden a vivir estresados, incrementando la posibilidad de que en su vida adulta presenten mayores dificultades para afrontar situaciones estresantes en distintas áreas de su vida (Palomar y Lanzagorta, 2005).

El apoyo social ha sido reconocido como un factor amortiguador frente a los estresores (Palomar y Cienfuegos, 2007). Los datos 
reportados por las familias participantes muestran que en promedio cuentan con tres familiares y/o amigos a los que se podría recurrir en busca de apoyo. La baja densidad y alto grado de homogeneidad es característica de las redes sociales deficientes (Sluzki, 1998) pero también debe entenderse desde la dinámica de la violencia intrafamiliar en donde el aislamiento hace a la perpetuación de las situaciones de maltrato y abuso (Hasanbegovic, 2016).

La asociación positiva entre nivel socioeconómico y percepción de apoyo social funcional ha sido establecida en otras investigaciones (Coppari et al., 2018; Rodríguez, 2010). Las personas en contextos de extrema pobreza y con historiales intergeneracionales de violencia, se vinculan con familias en similares condiciones lo que puede dificultar la disponibilidad de ayudas mutuas (Hasanbegovic, 2016) especialmente en lo que refiere al apoyo instrumental.

La correlación negativa (aunque moderada) entre estrés y apoyo social, también está en línea con datos obtenidos en otras investigaciones (Green y Rodgers, 2001; Guarino y Sojo, 2011; Palomar y Cienfuegos, 2007). Los individuos que tienen un alto apoyo social percibido generalmente evalúan su entorno como menos amenazador, facilitando una mejor lectura de las necesidades que propicia acciones de afrontamiento activas y efectivas (Lazarus y Folkman, 1986).

Los resultados de este estudio confirman, tristemente, la presencia y asociación entre variables que tornan difícil la superación de la problemática del maltrato. No obstante, existen una serie limitaciones que deben ser seńaladas. La muestra de participantes, aunque específica al tema en estudio, resulta acotada. Contar con una muestra de familias con maltrato infantil perteneciente a otros niveles socioeconómico seguramente hubiera colaborado a generar una mejor descripción de los aspectos que resultan característicos de las familias con maltrato infantil de nivel socioeconómico bajo. Los instrumentos utilizados presentan las limitaciones propias de los autoinformes, y además deberían ser adaptados al país.

A pesar de las limitaciones mencionadas, la descripción y análisis de las variables estudiadas ilustran una realidad que debe ser considerada 
en el diseño de las intervenciones psicosociales que pretendan modificaciones a la situación de maltrato infantil y el sostenimiento de esos cambios en el tiempo (Musitu y Cava, 2001; Minuchin et al., 2000).

Este trabajo pretende generar reflexión sobre la necesidad de ajustes en el diagnóstico incorporando la noción de polivictimización (Finkelhor et al., 2011) y acumulación de estresores (Cracco, 2017) para poder implementar acciones de trabajo coordinadas, interdisciplinarias e intersectoriales acordes a la situación de extrema vulnerabilidad. Modificar formas de vincularse para el ejercicio de una parentalidad bien tratante, requiere de una matriz de acciones e intervenciones que rebasen las esferas de lo individual y lo familiar, involucrando la red familiar extensa, a los individuos y servicios que componen la comunidad y el Estado (Barudy y Dantagnan, 2010; Gómez y Contreras, 2019; Gómez y Kotliarenco, 2010).

Desarrollar garantías de seguridad intrafamiliar a la infancia, construir contextos bien tratantes y seguros, conectados con las necesidades de los NNA, requiere de la moderación de los niveles de estrés, la satisfacción de necesidades básicas, inclusión en redes que incrementen los apoyos sociales brindando soporte emocional e instrumental efectivos (Barudy y Dantagnan, 2010; Gómez y Contreras, 2019; Gómez y Kotliarenco, 2010). Solo así se podrá romper con lógicas asistenciales que pueden vulnerar aún más a las familias (Minuchin et al., 2000) y que lamentablemente son reconocidas una y otra vez por los operadores psicosociales en el fenómeno de violencias intrafamiliares transgeneracionales.

\section{Referencias}

Bartholomae, S. y Fox, J. (2017). Economic stress and families. En C.

Price, K. Bush y S. Price (Eds.), Families and change (5 ${ }^{\text {th }}$ ed.), (pp. 341-358). Sage. 
Barudy, J. y Dantagnan, M. (2010). Los desafíos invisibles de ser madre o padre. Manual de evaluación de las competencias y la resiliencia parental. Gedisa.

Batthyány, K., Ferrari, F. y Scavino, S. (2015). Dichos y hechos: juventud, representaciones sobre el trabajo no remunerado y brechas en el uso del tiempo. En M. Boado (Coord.), El Uruguay desde la Sociología. Doble Clic.

Bentancor, G., Lozano, F. y Solari, M. (2017). Mapa de ruta para las situaciones de maltrato y abuso sexual en niños, niñas y adolescentes detectadas en el ámbito escolar. Unicef, Montevideo, Uruguay.

Boss, P. (2002). Family Stress Management: A contextual approach (2 $2^{\text {nd }}$ ed.). California: Sage.

Brooks-Gunn, J., Schneider, W. y Waldfogel, J. (2013). The Great Recession and the Risk for Child Maltreatment. Child abuse and neglect, 37(10), 721-729. https://doi.org/10.1016/j. chiabu.2013.08.004

Cabella, W., Fernández, M. y Prieto, V. (2015). Altas sociodemográfico y de la desigualdad en Uruguay. Trilce.

Cabrera, V., González, M. y Guevara, I. (2012). Estrés parental, trato rudo y monitoreo como factores asociados a la conducta agresiva. Universitas Psychologica, 11(1), 241-254.

Capano, A. y Pacheco, A. (2014). Estrés y violencia doméstica: un estudio con adultos referentes de nińos, niñas y adolescentes. Ciencias Psicológicas, 8(1), 31-42.

Carrasco, C. Borderías, T. y Torns, T. (2011). El trabajo de cuidados. Historia, teórica y politicas. Los libros de la catarata.

Casique, I. (2010). Factores de empoderamiento y protección de las mujeres contra la violencia. Revista Mexicana de Sociología, 72(1), 37-71.

Cleek, E., Wofsy, M., Boyd-Franklin, N., Mundy, B. y Howell, L. (2012). The Family Empowerment Program: an interdisciplinary approach to working with multi-stressed urban families. Family Process, 51(2), 207-217. http://dx.doi. $\mathrm{org} / 10.1111 / \mathrm{j} .1545-5300.2012 .01392 . x$ 
Conger, R., Conger, K., Elder, G., Lorenz, F., Simons, R. y Whitbeck, L. (1992). A Family Process Model of Economic Hardship and Adjustment of early adolescent boys. Child Development, 63(3), 526-541. http://dx.doi.org/10.2307/1131344

Conger, R., Conger, K. y Martin, M. (2010). Socioeconomic status, family processes, and individual development. Journal of Marriage y Family, 72(3), 685-704. http://dx.doi. org/10.1111/j.1741-3737.2010.00725.x

Convención de los Derechos del Niño. (1989). Recuperado de: https:// www.unicef.org/uruguay/spanish/CDN_20_boceto_final.pdf

Coppari, N., Bagnoli, L., Codas, G., López-Humada, H., MartínezCañete, Ú., Martínez, L. y Montanía, M. (2018). Relación entre apoyo social percibido y disposición resiliente en adolescentes paraguayos. Psychologia, 12(2), 13-22. https://doi. org/10.21500/19002386.3415

Cracco, C. (2017). Acumulación de estresores y funcionamiento familiar en contextos de pobreza. (Tesis doctoral). Universidad del Salvador, Argentina.

Cracco, C. y Blanco, M. (2015). Estresores y estrategias de afrontamiento en familias en las primeras etapas del ciclo vital y contexto socioeconómico. Ciencias Psicológicas, 9(2), 129-140.

Finkelhor, D., Turner, H. A., Hamby, S. L. y Ormrod, R. K. (2011). Polyvictimization: Children' Exposure to Multiple Types of Violence, Crime, and Abuse. Washington: Crimes Against Children Research Center. Recuperado de https://www.ncjrs.gov/pdffiles1/ojjdp/235504.pdf

García, M., Mateo, I. y Eguiguren, A. (2004). El sistema informal de cuidados en clave de desigualdad. Gaceta Sanitaria, 18(s/d), 132-139.

Gómez, E. y Contreras, L. (2019). Manual Escala de Parentalidad Positiva E2P V.2. Chile: Ediciones Fundación América por la Infancia. Recuperado de http://www.observaderechos.cl/site/ wp-content/uploads/2013/12/Manual-de-la-Escala-de-Parentalidad-Positiva.pdf 
Gómez, E. y Kotliarenco, M. (2010). Resiliencia familiar: un enfoque de investigación e intervención con familias multiproblemáticas. Revista de Psicologia, 19(2), 103-131. https://doi.org/10.5354/0 719-0581.2010.17112

Green, B. y Rodgers, A. (2001). Determinants of social support among low-income mothers: A longitudinal analysis. American Journal of Comunitary Psychology, 29(3), 419-442.

Grinberg, J. (2015). Entre la pediatría, el psicoanálisis y el derecho: apuntes sobre la recepción, reelaboración y difusión del "maltrato infantil" en Argentina. Revista de Estudios Sociales, 1(53), 77-89. http://dx.doi.org/10.7440/res53.2015.06

Guarino, L. y Sojo, V. (2011). Apoyo social como moderador del estrés en la salud de los desempleados. Universitas Psychologica, 10(3), 867-879.

Hasanbegovic, C. (2016). Violencia basada en el género y el rol del Poder Judicial. Revista de la Facultad de Derecho, 40(sd), 119-158. https://doi.org/10.22187/rdf201616

Hernández-Córdoba, A. (1991). Estrés en la familia colombiana: Tensiones tipicas y estrategias de afrontamiento. Bogotá, Universidad Santo Tomás.

Hill, S. (2012). Families. A social class perspective. Sage.

Intebi, I. (2009). Intervención en casos de maltrato infantil. España: Dirección General de Políticas Sociales, Gobierno de Cantabria. Recuperado de https://www.serviciossocialescantabria.org/uploads/documentos\%20e\%20informes/ Intervencion $\% 20$ en $\% 20$ casos $\% 20 \mathrm{de} \% 20$ maltrato $\% 20$ infantil.\%20Noviembre\%202009.pdf

Kaztman, R., y Rodríguez, F. (2005). Las formas de constitución de las familias pobres urbanas en Uruguay: consecuencias sobre el rendimiento educativo de los niños. Prisma, 21(s/d) 117-139.

Khaw, L. (2017). Stress and coping with intimate partner violence. En C. Price, K. Bush y S. Price (Eds.), Families and change ( $5^{\text {th }}$ ed.), (pp. 249-268). Sage. 
Kiernan, K. y Huerta, M. (2008). Economic deprivation, maternal depression, parenting and children's cognitive and emotional development in early childhood. British Journal of Sociology, 59(4), 783-806. http://dx.doi. org/10.1111/j.1468-4446.2008.00219.x

Lazarus, R. y Folkman, S. (1986). Estrés y procesos cognitivos. Barcelona: Martínez Roca.

Leinonen, J., Solantaus, T. y Punamäki, R. (2002). The specific mediating paths between economic hardship and the quality of parenting. International Journal of Behavioral Development, 26(5), 423-435. http://dx.doi.org/10.1080/01650250143000364

Ley $\mathrm{N}^{\circ}$ 17.823. (2004). Código de la niñez y la adolescencia del Uruguay. Recuperado de https://www.impo.com.uy/bases/ codigo-ninez-adolescencia/17823-2004

Masarik, A., y Conger, R. (2017). Stress and child development: a review of the Family Stress Model. Current Opinion in Psychology, 13(s/d), 85-90. http://dx.doi.org/10.1016/j. copsyc.2016.05.008

McCubbin, H., Patterson, J. y Wilson, L. (1989). Inventario Familiar de Eventos y Cambios Vitales. En D. Olson, H. McCubbin, H. Barnes, A. Larsen, M. Muxen, y M. Wilson, Family Inventories, University of Minnesota, Minnesota. Traducción de A. Hernández, Inventarios sobre familia, (pp. 127-159). Universidad de Santo Tomás, Bogotá.

Minuchin, P., Colapinto, J. y Minuchin, S. (2000). Pobreza, institución, familia. Amorrortu.

Montero, I. y León, O. (2005). Sistema de clasificación del método en los informes de investigación en Psicología. International Journal of Clinical and Health Psychology, 5(1), 115-127.

Musitu, G. y Cava, M. (2001). La familia y la educación. Octaedro.

Newland, R., Crnic, K., Cox, M. y Mills-Koonce, R. (2013). The family model stress and maternal psychological symptoms: mediated pathways from economic hardship to parenting. Journal of 
Family Psychology, 27(1), 96-105. https://doi.org/10.1037/ a0031112

Organización de las Naciones Unidas. (2020). La mitad de los niños sufren algún tipo de maltrato físico, sexual o psicológico cada año. Noticias ONU. Recuperado de https://news.un.org/es/ story/2020/06/1476222

Palomar, J. y Cienfuegos, Y. (2007). Pobreza y apoyo social: un estudio comparativo en tres niveles socioeconómicos. Revista Interamericana de Psicología, 41(2), 177-188.

Palomar, J. y Lanzagorta, N. (2005). Pobreza, recursos psicológicos y movilidad social. Revista Latinoamericana de Psicología, 37(1), $9-45$.

Perera, M. (2018). Índice de Nivel Socioeconómico. Montevideo: CINVE. Recuperado de https://portal.factum.uy/pdf/INSE_ informe_2018.pdf

Rodríguez, E. (2010). Relación entre nivel socioeconómico, apoyo social percibido, género y depresión en niños. Interdisciplinaria, 27(2), 261-275.

Rodríguez, S. y Enrique, H. (2007). Validación argentina del Cuestionario MOS de Apoyo Social Percibido. Psicodebate, 7(12), 155-168. https://doi.org/10.18682/pd.v7i0.433

Sastre, A. (2015). Más solas que nunca. La pobreza infantil en familias monoparentales. España: Save The Children. Recuperado de: https://www.savethechildren.es/sites/default/files/imce/docs/ mas_solas_que_nunca.pdf

Sherbourne, C. y Stewart, A. (1991). The MOS Social Support Survey. Social Science and Medicine, 32(6),705-714. https://doi. org/10.1016/0277-9536(91)90150-B

Sistema Integral de Protección a la Infancia y a la Adolescencia contra la Violencia. (2020). Informe de Gestión 2019. Recuperado de http://www.inau.gub.uy/novedades/noticias/item/2934-sipiaven-cifras-se-presento-el-informe-de-gestion-2019.

Sluzki, C. (1998). La red social: frontera de la práctica sistémica. Barcelona: Gedisa. 
Waldfogel, J., Craigie, T. y Brooks-Gunn, J. (2010). Fragile families and child wellbeing. The Future of Children, 20, 87-112. http:// dx/doi.org/10.1353/foc.2010.0002

Recibido: 2020-12-23

Revisado: 2021-08-01

Aceptado: 2021-08-02 